\title{
Introduction to data collection for performance analysis and their integration in martial arts training. A biomechanical and motor control perspective
}

\author{
Marco A. C. BRANCO ${ }^{*}$, Gonçalo A. C. BRANCO ${ }^{1}$, \& António VENCESBRIT01,2,3,4 \\ ${ }^{1}$ Sports Sciences School of Rio Maior - Polytechnic Institute of Santarém (Portugal) \\ ${ }^{2}$ Life Quality Research Centre - CIEQV (Portugal) \\ ${ }^{3}$ Research Unit of the Polytechnic Institute of Santarém - UIIPS (Portugal) \\ ${ }^{4}$ International Martial Arts and Combat Sports Scientific Society - IMACSSS (Poland)
}

5th IMACSSS World Scientific Congress Abstracts, Rio Maior (Portugal), October 6-8 Type: Workshop

\section{Introduction}

In order to improve the development of functional, physical, technical and tactical skills of martial arts athletes, coaches look for new ways to collect data. One of the most frequent methods is kinematic analysis, which allows spatial and temporal data to be collected.

The most common method of kinematic analysis is videography, which allows data collection with affordable equipment to the general public. Video cameras are used regularly as they are a quick way to obtain information not available to the naked eye and, therefore, an essential component to the training of combat sports athletes as well as martial arts practitioners. Videography allows a reliable data collection technique since the cameras positioning and calibration are met. The camera features should be taken into account for a better analysis, including image quality, capture frame rate, shutter speed, lens aperture and form of synchronization between cameras. The capture frame rate is related to the technical gesture execution speed, which can be found in Payton and Bartlett (2007).

The first step to consider is the number of cameras to use, which directly impacts the data we want to gather: two-dimensional (2D), for which we need at least one camera; or three-dimensional (3D), which takes at least two video cameras. On a 2D analysis, the camera must be placed perpendicular to the movement plane that is the focus of the analyses. On a three-dimensional analysis, cameras must be placed perpendicular to at least two movement planes and, therefore, conduct a $90^{\circ}$ angle between them (Bartlett, 2007).

After setting the camera location, spatial references should be placed, which will later be added into the kinematics analysis software, commonly called calibration volume. This volume is recorded for a few seconds, after which cameras cannot be moved, since there is a risk of losing calibration. Calibration through spatial references allows the conversion of segments or anatomical markers from the image (whose units are the pixels) into metric units (the SI units for length) by the direct linear transformation (DLT) algorithm (Abdel-aziz \& Karara, 1971). The technique must be recorded in a way that allows the identification of the anatomical markers which in turn enable to create a biomechanical model.

Winter, Patla, Prince, Ishac and Gielo-Perczak (1998) developed a 14 segment model with the purpose of estimate the total body center of mass, which needs 21 predefined markers, further the International Society of Biomechanics (ISB) and several other authors defined the placement of those anatomical markers in a way that allows the movement analysis either linear or angular (Cappozzo, Catani, Della Croce, \& Leardini, 1995; de Leva, 1996; Robertson, Caldwell, Hamill, Kamen, \& Whittlesey, 2014; Wu, et al., 2002, 2005).

*Email: marcobranco@esdrm.ipsantarem.pt

(C) 2016 Universidad de León. www.unileon.es 
A variety of open source software has been developed in the recent years, enabling a more affordable kinematic analysis to coaches and investigators. Two of those are Kinovea and Skillspector. The first one only allows for 2D analysis, while the other allows for both 2D and 3D, but increasing its complexity. Tutorials and videos for these programs exist online explaining some analysis procedures. After recording the calibration volume and the movement itself, we proceed to identify the markers on the calibration volume, defining its XYZ coordinates, as well as identifying the anatomical markers in the video of the technique being executed. This protocol allows the data collection of either spatial or temporal data for analysis. Acknowledging that with time and position data, there are several variables that can be determined related to anatomical markers and body segments, the possible information that can be extracted are endless. Most of the available software allows extracting, in addition to the execution time and the position of the body segments, variables such as position, velocity, and acceleration, both for linear and angular data.

In the recent years, many studies showed us several performance indicators which combined with coaches' formation, are a potential performance developer for combat sports and martial arts athletes. Some kinematic performance indicators were recently studied. Loturco, Artioli, Kobal, Gil and Franchini (2014) found that the auto-selected distance, the maximum strength and the lower and superior body power are factors that affects the acceleration of a punch. Bolander, Neto and Bir (2009) found that the portion of the hand which makes the contact influences the impact forces of the punch, also when the attack occurs to the thorax. The same authors, show that larger distance to the target achieve higher acceleration as well as the ability to aim further behind the target. However, these indicators need a good technical performance as Neto, Marzullo, Bolander and Bir (2013) determined. According to them, expert athletes achieve higher hand acceleration and precision. VencesBrito et al. (2014) determined that while performing of a frontal kick, expert athletes achieved lower articular range with early velocity peaks than their inexperienced counterparts.

Although less studied in combat sports and martial arts, other data analysis methods can be performed, like the study of synchronization (Richardson, Marsh, Isenhower, Goodman, \& Schmidt, 2007; Schmidt, Fitzpatrick, Caron, \& Mergeche, 2011) between athletes, or non-linear analysis like recurrence quantification.

\section{References}

Abdel-aziz, Y. I., \& Karara, H. M. (1971). Direct linear transformation from comparator coordinates into object space coordinates in close-range photogrammetry. Proceedings of the ASP/UI Symposium on Close-Range Photogrammetry (Vol. 1, pp. 1-18). Falls Church, VA: American Society of Photogrammetry.

Bartlett, R. (2007). Introduction to sports biomechanics: analysing human movement patterns (2nd ed.). Milton Park, Abingdon, Oxon ; New York, NY: Routledge.

Bolander, R. P., Neto, O. P., \& Bir, C. A. (2009). The effects of height and distance on the force production and acceleration in martial arts strikes. Journal of Sports Science and Medicine, 8 (CSSI3), 47-52.

Cappozzo, A., Catani, F., Della Croce, U., \& Leardini, A. (1995). Position and Orientation in-Space of Bones during Movement - Anatomical Frame Definition and Determination. Clinical Biomechanics, 10(4), 171-178.

de Leva, P. (1996). Adjustments to Zatsiorsky-Seluyanov's segment inertia parameters. Journal of Biomechanics, 29(9), 1223-1230.

Loturco, I., Artioli, G. G., Kobal, R., Gil, S., \& Franchini, E. (2014). Predicting Punching Acceleration from Selected Strength and Power Variables in Elite Karate Athletes: A Multiple Regression Analysis. Journal of Strength and Conditioning Research, 28(7), 1826-1832.

Neto, O. P., Marzullo, A. C. D., Bolander, R. P., \& Bir, C. A. (2013). Martial arts striking hand peak acceleration, accuracy and consistency. European Journal of Sport Science, 13(6), 653-658.

Payton, C., \& Bartlett, R. (2007). Biomechanical evaluation of movement in sport and exercise: the British Association of Sport and Exercise Sciences guide. Abingdon, Oxon; New York, NY: Routledge. 
Richardson, M. J., Marsh, K. L., Isenhower, R. W., Goodman, J. R. L., \& Schmidt, R. C. (2007). Rocking together: Dynamics of intentional and unintentional interpersonal coordination. Human Movement Science, 26(6), 867-891.

Robertson, D. G. E., Caldwell, G. E., Hamill, J., Kamen, G., \& Whittlesey, S. N. (2014). Research Methods in Biomechanics (2nd. ed.). Champaign, IL, USA: Human Kinetics.

Schmidt, R. C., Fitzpatrick, P., Caron, R., \& Mergeche, J. (2011). Understanding social motor coordination. Human Movement Science, 30(5), 834-845.

VencesBrito, A. M., Branco, M. A. C., Fernandes, R. M. C., Ferreira, M. A. R., Fernandes, O. J. S. M., Figueiredo, A. A. A., \& Branco, G. (2014). Characterization of kinesiological patterns of the frontal kick, mae-geri, in karate experts and non-karate practitioners. Revista de Artes Marciales Asiaticas, 9(1), 20-31.

Winter, D. A., Patla, A. E., Prince, F., Ishac, M., \& Gielo-Perczak, K. (1998). Stiffness control of balance in quiet standing. Journal of Neurophysiology, 80(3), 1211-1221.

Wu, G., Siegler, S., Allard, P., Kirtley, C., Leardini, A., Rosenbaum, D., . . . Stokes, H. (2002). ISB recommendation on definitions of joint coordinate system of various joints for the reporting of human joint motion - part 1: ankle, hip, and spine. Journal of Biomechanics, 35(4), 543-548.

Wu, G., van der Helm, F. C. T., Veeger, H. E. J., Makhsous, M., Van Roy, P., Anglin, C., . . Buchholz, B. (2005). ISB recommendation on definitions of joint coordinate systems of various joints for the reporting of human joint motion - Part II: shoulder, elbow, wrist and hand. Journal of Biomechanics, 38(5), 981-992.

Key words: Data collection; biomechanics; combat sports. 